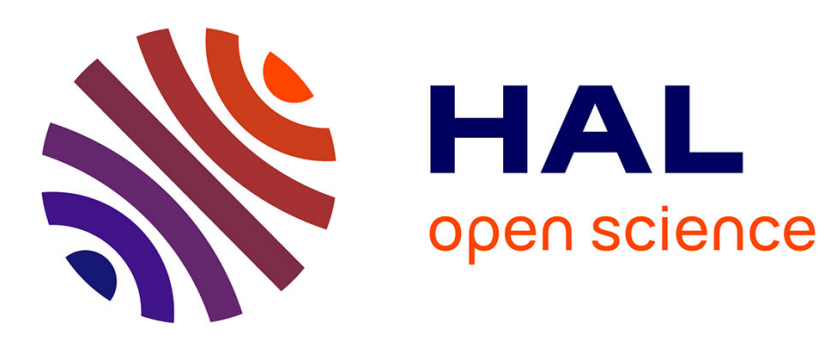

\title{
Stability analysis of rough surfaces in adhesive normal contact
}

\author{
Valentine Rey, Jeremy Bleyer
}

\section{To cite this version:}

Valentine Rey, Jeremy Bleyer. Stability analysis of rough surfaces in adhesive normal contact. Computational Mechanics, 2018, 10.1007/s00466-018-1556-y . hal-01731280

\section{HAL Id: hal-01731280 \\ https://hal-enpc.archives-ouvertes.fr/hal-01731280}

Submitted on 14 Mar 2018

HAL is a multi-disciplinary open access archive for the deposit and dissemination of scientific research documents, whether they are published or not. The documents may come from teaching and research institutions in France or abroad, or from public or private research centers.
L'archive ouverte pluridisciplinaire HAL, est destinée au dépôt et à la diffusion de documents scientifiques de niveau recherche, publiés ou non, émanant des établissements d'enseignement et de recherche français ou étrangers, des laboratoires publics ou privés. 


\title{
Stability analysis of rough surfaces in adhesive normal contact
}

\author{
Valentine Rey · Jeremy Bleyer
}

Received: date / Accepted: date

\begin{abstract}
This paper deals with adhesive frictionless normal contact between one elastic flat solid and one stiff solid with rough surface. After computation of the equilibrium solution of the energy minimization principle and respecting the contact constraints, we aim at studying the stability of this equilibrium solution. This study of stability implies solving an eigenvalue problem with inequality constraints. To achieve this goal, we propose a proximal algorithm which enables qualifying the solution as stable or unstable and that gives the instability modes. This method has a low computational cost since no linear system inversion is required and is also suitable for parallel implementation. Illustrations are given for the Hertzian contact and for rough contact.
\end{abstract}

Keywords Adhesive normal contact · BEM · rough surfaces · Stability

\section{Introduction}

Both natural and manufactured surfaces, even if they appear flat from a macroscopic viewpoint, are actually composed of many asperities. The size of those asperities depends on the material and on the way the surface was created or manufactured and usually covers many scales. Indeed, rough surfaces are often modeled as self-affine surfaces with a rich spectrum of wavelengths [26]. Contact mechanics of rough surfaces aims at accounting for the complex interactions between these asperities. Applications are numerous and include friction, wear, or the thermal or electrical conductivity of an interface.

V. Rey,

Laboratoire de simulation en mécanique des solides, Institut ENAC

Ecole Polytechnique Fédérale de Lausanne (EPFL)

Station Z

CH-1015 Lausanne

E-mail: valentine.rey@epfl.ch

J. Bleyer,

Ecole des Ponts ParisTech, Laboratoire Navier (ENPC-IFSTTAR-CNRS UMR 8205)

Université Paris-Est

6-8 av. Blaise Pascal, Cité Descartes

77455 Champs-sur-Marne, FRANCE

E-mail: jeremy.bleyer@enpc.fr

Corresponding author: Valentine Rey 
Because of the multiscale nature of the rough surfaces, it is necessary to introduce the relevant physics at the micro scale. Indeed, at the small scale, adhesion is not negligible and may strongly change the morphology of contact clusters or global contact area with consequences on leakage properties for example. Note that one of the consequences of the adhesive behaviour is the possibility of unstable solutions illustrated by snap-back instabilities. It is a major challenge for the micro-electromechanical systems (MEMS) and nano-electromechanical systems (NEMS). This is one of the reason why adhesive contact between rough surfaces has been addressed. In the 1970's, adhesion was first introduced into the classical Hertz contact. In [24,21,12], the authors consider one unique spherical asperity or flat-ended sphere in [25]. The analytical approach enables to perfom a complete study : from the computation of the equilibrium solution to the stability of those solutions. In order to describe more realistic roughnesses, periodic sinusoidal roughness [18] or multi-asperity adhesive contact problems have been studied [33]. More recently, adhesive contact was investiagted in the case of a rough punch with a main curvature and additional roughness (through power-law graded material in [17], using the small scale topography of the punch in [22] or combining it with multi-asperity [40]). Once again, the analytical approach enables deriving the complete study up to the hysteresis cycles. Unfortunately, those geometries rely on spherical periodicity and they imply separation of length scales, which is not verified for many rough surfaces [26].

Using tools to generate large rough surfaces, numerical simulations offer the possibility to compute the solution of adhesive contact without neglecting elastic interactions on a representative roughness. These computations can be performed with the Boundary Element Method (BEM)[1-3] and the Green's Function Molecular Dynamics method (GFMD) [27,32].

As mentioned earlier, the consequence of adhesive behavior is the possible existence of unstable equilibrium solutions. Indeed, adhesion is known to conduct to snap-back instabilities. This question is often disregarded in publications based on numerical simulations that focus on obtaining an equilibrium solution, which may already be difficult. Nonetheless some publications $[24,4,25,27,30]$ study stability issues in the case of the Maugis-Dugdale [24] model for adhesion. This model considers constant adhesion force so that the adhesive zone can be seen as a cohesive zone. However, those studies are limited to simple profiles of surface (one indenter) for which analytical solutions are available, which eases the stability analysis.

The objective of this article is the stability analysis of equilibrium solutions for adhesive frictionless normal contact between rough surfaces. We consider one elastic flat semi infinite solid and one rigid rough semi infinite solid. Using integral formulation [34], the contact problem is written only at the surface of the elastic solid. The equilibrium solution is computed using a FFT-based BEM algorithm [36]. In this article, we will not consider plasticity nor friction. Indeed, although those phenomena are expected to take place in rough contact, including them in the model would involve adding new non-linearities and bring major difficulties to the problem.

Stability analysis usually consists in studying perturbations around an equilibrium solution [29]. In presence of contact, difficulty arises since all perturbations are not admissible because some part of the surfaces being in contact. Indeed, perturbations leading to inter-penetration must not be considered. As a consequence, the stability analysis in case of contact can be formulated as a cone-constrained eigenvalue problem [37,9,35,20,10,11]. Exploiting algorithms used in optimization, we propose a systematic approach to study the stability of an equilibrium solution in presence of contact. This approach is independent from the adhesive potential and from the geometry of the surface. It enables qualifying the solution as stable or unstable. Moreover, in case the solution is unstable, the computed eigenvectors give the instability modes. One of the advantage is that this method does not require to solve any additional contact elastic problem or linear system: it is composed only of local operations which can be easily done in parallel. 

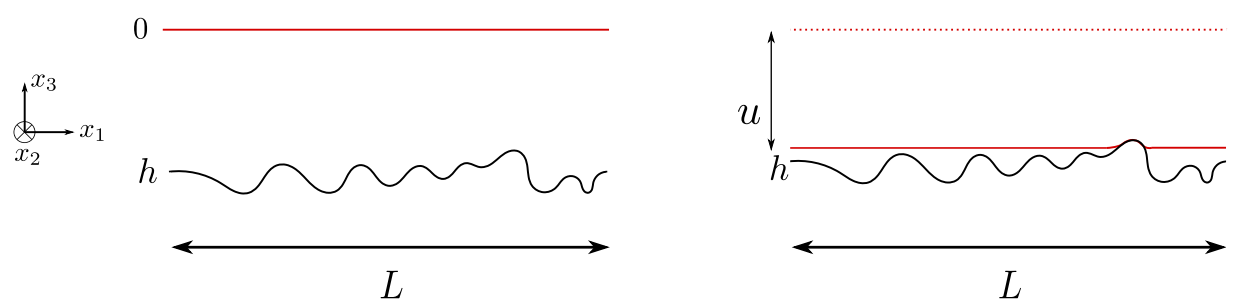

Fig. 1: A sketch of two solids coming into contact (2d view).

The paper is organized as follows. The definition of adhesive frictionless normal contact mechanical problem with periodic surfaces and the numerical method used to solve it are described in Section 2. In Section 3, we detail the stability criterion in case of contact and propose an algorithm computing the smaller eigenvalue of the constrained system. Finally, in Section 4, we give several numerical illustrations.

\section{Adhesive normal contact with BEM}

We study the frictionless normal contact between two homogeneous semi-infinite solids: one flat elastic isotropic deformable solid (under small displacements hypothesis) of Young's modulus $E$ and Poisson's ratio $\nu$ and one rigid (infinitely stiff) rough surface with the height profile $h$ defined by $\underline{x} \rightarrow x_{3}=h(\underline{x})$.

On Figure 1, we give a sketch of two solids of size $L$ : one rigid rough surface with the height profile $h(\underline{x})=h\left(x_{1}, x_{2}\right)$ and a flat elastic surface whose vertical displacement is denoted by $u(\underline{x})$. The origin of the system of axes can be chosen arbitrary since both solids are semi infinite in the third direction. On this sketch, both $h$ and $u$ are negative.

\subsection{Continuous problem}

Integral formulation (see [3] or more generally [16,13] with elastoplastic behaviour) enables rewriting the three dimensional contact problem set on the semi infinite solid only at the normal surface $S$ of this solid. It is attractive from a computational viewpoint since it reduces the dimension of the problem. Once this problem is solved, the surface quantities, such as the surface displacement or the pressure, can be used to determine the state of the bulk below the surface. Indeed, both strain and stresses in the volume can be computed as a post-processing step.

The elastic behavior is described through a convolution product with a kernel defined from a fundamental solution. More precisely, the link between normal displacement $u(\underline{x})$ and the normal pressure $p(\underline{x})$ at the surface is:

$$
u(\underline{x})=\int_{S} K\left(\underline{x}-\underline{x}^{\prime}\right) p(\underline{x}) d \underline{x}^{\prime}
$$

If we use the Boussinesq's fundamental solution [19], $K(\underline{x})$ is the surface deflection at the point of coordinate $\underline{x}$ produced by a concentrated normal load of unit magnitude, applied on the surface at the origin $(0,0)$. In this paper, we decide to use the Westergaard's solution [39] that connects pressure to displacements in Fourier's space thanks to influence coefficients (see section 2.2). In any case, the elastic energy density $w_{e l}(u)=p(u) u / 2$ is a quadratic function of the surface displacement $u$ and $p(u)=d w_{e l} / d u$. 
We note $g$ the so-called gap, which is the distance between the two surfaces and which is defined by:

$$
g(\underline{x})=u(\underline{x})-h(\underline{x})
$$

The condition of non interpenetration between the two solids imposes that:

$$
\forall \underline{x}, g(\underline{x}) \geqslant 0
$$

The prescribed loading is given in terms of the mean value of the gap:

$$
\frac{1}{\operatorname{mes}(S)} \int_{S}(u(\underline{x})-h(\underline{x})) d S=g_{0}
$$

When adhesion is considered, the potential energy $E_{p}$ contains an additional contribution of adhesive energy $E_{a d h}$ in addition to the elastic energy $E_{e l}$ :

$$
E_{p}=E_{\text {elas }}+E_{a d h}=\int_{S} w_{e l}(u(\underline{x})) d S+\int_{S} f(g(\underline{x})) d S
$$

where the adhesive energy density $f(g)$ is a function of the distance $g$ between the two surfaces. It is defined only for positive values of $g$ and it is assumed to be at least twice differentiable on its domain of definition so that the associated second derivative is well defined when performing the stability analysis.

The displacement field $u$ is the solution of the minimization problem:

$$
u=\underset{u \in \mathcal{A}}{\operatorname{argmin}} E_{p}(u)
$$

where $\mathcal{A}$ is the space of admissible displacement fields characterized by:

$$
u \in \mathcal{A} \Leftrightarrow \forall x, u(\underline{x})-h(\underline{x}) \geqslant 0 \text { and } \frac{1}{\operatorname{mes}(S)} \int_{S}(u(\underline{x})-h(\underline{x})) d S=g_{0}
$$

Let us define the Lagrangian of the system:

$$
\mathcal{L}(u, \mu)=\int_{S}\left(w_{e l}(u)+f(g)\right) d S-\int_{S} \mu(u-h) d S=E_{p}(u)-\int_{S} \mu(u-h) d S
$$

where $\mu$ is the positive Lagrange multiplier associated to the contact constraint: $\mu \geqslant 0$. The stationarity of the Lagrangian reads:

$$
\frac{\partial \mathcal{L}}{\partial u}(u, \mu)=\frac{d w_{e l}}{d u}+\frac{d f}{d u}-\mu=p+\frac{d f}{d u}-\mu=0
$$

Therefore, the Lagrange multiplier is equal to the sum of the elastic $(p)$ and adhesive $(d f / d u)$ forces. Orthogonality for adhesive contact reads:

$$
\forall \underline{x}, \quad\left\{\begin{array}{l}
\left(p(\underline{x})+\frac{d f}{d u}\right) g(\underline{x})=0 \\
p(\underline{x})+\frac{d f}{d u} \geqslant 0 \\
g(\underline{x}) \geqslant 0
\end{array}\right.
$$

We can give a mechanical interpretation of the first equality, which is the orthogonality between the constraint and the associated Lagrange multiplier. On the contact zone $(g=0)$, the total force is strictly positive (the sum of adhesive forces and elastic forces is a compressive force). On 


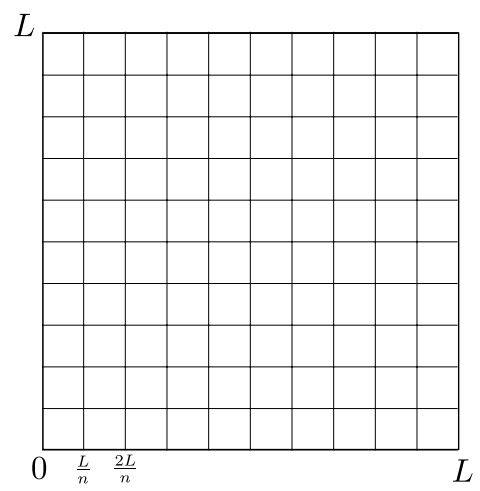

Fig. 2: Grid used for the discretization of the contact problem on the square surface $\Omega=[0 ; L] \times$ $[0 ; L]$

the non contact zone $(g>0)$, the total force is null (balance of elastic and adhesive forces).

Let us call equilibrium solution a critical point of the problem (6). Without adhesion, the total energy is equal to the elasticity energy $E_{\text {elas }}$, which is a convex function of the surface displacement so that the critical point is unique and is the global minimizer of the problem. However, in presence of adhesion, the total energy usually becomes non-convex and a critical point is not necessarily a global, let alone a local, minimizer but may also characterize a saddle point which corresponds to an unstable equilibrium position as discussed in Section 3.

\subsection{Discretized problem}

Instead of a semi infinite surface, we consider a square surface of length $L$ with periodic boundary conditions (PBC). Those PBC ensure that the height profile is such that $h(0, x)=h(L, x)$, $h(x, 0)=h(x, L)$ for all $x=0, l . . L$. On Figure 1, one can observe that the rough profile is periodic in the sense that $h(0)=h(L)$. One can easily define the finite size continuous problem derived from (6). To solve this problem, the square surface $\Omega=[0 ; L] \times[0 ; L]$ is discretized as a uniform grid with discretization length $l$. We aim at solving the discrete problem on the $N=n^{2}=\left(\frac{L}{l}\right)^{2}$ grid points (collocation) using BEM [3], as illustrated on Figure 2 .

We will denote by bold font $\mathbf{u}$ the vector collecting the $N$ nodal values of the normal surface displacement $u$ and $\mathbf{p}$ the vector collecting the $N$ nodal values of the associated normal pressure. The scalar $u_{i}$ is the value of the vertical displacement at the grid node $i$. We denote by e the vector with all components equal to 1 .

The convolution product (1) is computed in Fourier's space. Therefore, one can compute the displacement $\mathbf{u}$ from the pressure $\mathbf{p}$ :

$$
\mathbf{u}=\mathrm{FFT}^{-1}(\mathbf{W} \operatorname{FFT}(\mathbf{p}))
$$

where FFT is the 2D Fast Fourier Transform, FFT $^{-1}$ is the Backward 2D Fast Fourier Transform, and $\mathbf{W}$ is a diagonal $N \times N$ matrix that contains the influence coefficients for the reference Westergaard solution [39]. These constant coefficients depend on the discrete set of frequencies admissible for the chosen discretization and on the Young's modulus $E$ (see [38] for their expressions). In $\mathbf{W}$, the coefficient associated to the 0 -frequency is 0 . Indeed, the displacement of the 
surface is only known up to a rigid-body motion. It is possible to obtain a pressure $\mathbf{p}$ with mean value 0 from the displacement $\mathbf{u}$ using the equality:

$$
\mathbf{p}=\mathrm{FFT}^{-1}\left(\mathbf{W}^{-\mathbf{1}} \mathrm{FFT}(\mathbf{u})\right)=\mathbf{K u}
$$

where $\mathbf{K}$ is the corresponding linear operator which is a dense matrix. The obtained pressure is such that its mean is 0 because the coefficient of $\mathbf{W}^{-\mathbf{1}}$ associated to the 0 -mode is 0 .

We define the gap vector $\mathbf{g}=\mathbf{u}-\mathbf{h}$ and the admissible space as:

$$
\mathbf{u} \in \mathcal{A} \Leftrightarrow \forall i \in\{1, \ldots, N\}, g_{i} \geqslant 0 \text { and } \frac{1}{N} \sum_{i=1}^{N} g_{i}=\frac{1}{N} \mathbf{e}^{T} \mathbf{g}=g_{0}
$$

The discretized problem reads:

$$
\text { Find } \mathbf{u} \in \mathcal{A} \text { minimizing } E_{p}(\mathbf{u})
$$

where:

$$
E_{p}(\mathbf{u})=\frac{1}{2} \mathbf{u}^{T} \mathbf{K u}+\sum_{i=1}^{N} f\left(g_{i}\right)
$$

Using a conjugate gradient algorithm with projections [36], we can compute an equilibrium solution of problem (14). Both displacement and pressure fields are periodic. In the context of adhesive contact, the potential energy to minimize is not convex so that several local minimizers may exist. Therefore, it is possible to reach different equilibrium solutions depending on the initialization of the descent algorithm.

\section{Stability analysis}

Characterizing the stability of an equilibrium position is important to assess the reliability of the computed solution since an unstable solution will be sensitive to small imperfections. In the case of adhesive contact, it can be expected that equilibrium positions will be stable if adhesion effects are negligible with respect to elasticity (e.g. low surface energy/very short range interaction). However, as the surfaces become closer and closer, adhesive effects become more important and may lead to snap-on/snap-off types of instabilities, that is sudden contact (resp. sudden detachment) of part of the elastic surface. The proposed stability analysis will aim at determining if a computed equilibrium solution is stable and, if not, will exhibit the associated instability mode.

\subsection{Stability analysis with normal contact}

We recall here the results obtained in [5] regarding the stability of elastic bodies in normal contact applied to the present problem. Let us first define the set $V_{0}$ of virtual velocity fields $\delta v$ which are periodic and with zero mean value:

$$
V_{0}=\left\{\delta v \text { periodic and } \int_{S} \delta v(\underline{x}) d S=0\right\}
$$

Usually, in the absence of contact constraints, the stability analysis of conservative system around an equilibrium position requires to study the sign of the total energy second derivative (Lejeune-Dirichlet criterion [29]) for a set of kinematically admissible perturbations $\delta v \in V_{0}$ : 


$$
\delta v \cdot \frac{\partial^{2} E_{p}}{\partial u^{2}}\left(u_{e q}, \lambda\right) \cdot \delta v>0 \quad \forall \delta v \in V_{0} \Rightarrow \text { stable equilibrium }
$$

where $u_{e q}$ is the equilibrium configuration we aim at studying and $\lambda$ a loading parameter that is fixed (for instance the average gap $g_{0}$ ).

In case of contact, the stability study is more difficult since we need to take into account the contact constraints. In the case of frictionless contact with a rigid surface, the contact reactions do not create mechanical work. As a consequence, the space of admissible perturbations is modified by adding the contact constraint [5]. The new set of kinematically admissible perturbations is:

$$
V_{0}^{+}=\left\{\delta v \in V_{0} \text { and such that } \delta v(\underline{x}) \geqslant 0 \forall \underline{x} \in I_{c}\right\}
$$

where $I_{c} \subset \Omega$ is the contact zone in the equilibrium configuration:

$$
\underline{x} \in I_{c} \Leftrightarrow u_{e q}(\underline{x})-h(\underline{x})=0
$$

This new set ensures that perturbations do not create interpenetration. At equilibrium, the associated Lagrange multiplier $\mu_{e q}$ is therefore such that $\mu_{e q}(\underline{x}) \geqslant 0$ for $\underline{x} \in I_{c}$ and $\mu_{e q}(\underline{x})=0$ otherwise.

The directional derivative of the Lagrangian around the equilibrium position $\left(u_{e q}, \mu_{e q}\right)$ for an admissible perturbation direction $\delta v$ is given by:

$$
\left.\frac{d \mathcal{L}}{d t}\left(u_{e q}+t \delta v, \mu_{e q}, \lambda\right)\right|_{t=0}=\frac{\partial \mathcal{L}}{\partial u}\left(u_{e q}, \mu_{e q}, \lambda\right) \cdot \delta v
$$

which gives the optimality condition (9) characterizing the equilibrium position when equating this derivative with zero. The second directional derivative is then given by:

$$
\left.\frac{d^{2} \mathcal{L}}{d t^{2}}\left(u_{e q}+t \delta v, \mu_{e q}, \lambda\right)\right|_{t=0}=\delta v \cdot \frac{\partial^{2} \mathcal{L}}{\partial u^{2}}\left(u_{e q}, \mu_{e q}, \lambda\right) \cdot \delta v=\delta v \cdot \frac{\partial^{2} E_{p}}{\partial u^{2}}\left(u_{e q}, \lambda\right) \cdot \delta v \quad \forall \delta v \in V_{0}^{+}
$$

Now, since $\mathcal{L}\left(u(t), \mu_{e q}\right) \leqslant E(u(t))$ with $u(t)=u_{e q}+t \delta v$ for all $t \geqslant 0$, a local minimum of the energy at equilibrium is ensured if the previous second derivative is positive for all admissible perturbations. The stability criterion then reads:

$$
\delta v \cdot \frac{\partial^{2} E_{p}}{\partial u^{2}}\left(u_{e q}, \lambda\right) \cdot \delta v>0 \quad \forall \delta v \in V_{0}^{+} \Rightarrow \text { stable equilibrium }
$$

The objective of the present work is to qualify the equilibrium solution obtained by the contact algorithm as either stable or unstable using the previous criterion. To do so, a first approach would be to compute the smallest eigenvalue of the energy second derivative around the equilibrium solution. If the associated eigenvector satisfies the contact constraints imposed by $V_{0}^{+}$, the stability of the equilibrium solution can be directly deduced from the sign of the eigenvalue. However, if this eigenvector does not belong to $V_{0}^{+}$, conclusion on the stability of the equilibrium position is not possible. In this case, one has to compute the minimum eigenvalue of the energy second derivative under the constraint that the eigenvector has to belong to $V_{0}^{+}$. This eigenvalue can be obtained as the solution to the following constrained Rayleigh-quotient problem: 


$$
\begin{gathered}
\lambda_{\min }=\min _{\delta v} \frac{1}{2} \delta v \cdot \frac{\partial^{2} E_{p}}{\partial u^{2}}\left(u_{e q}, \lambda\right) \cdot \delta v \\
\text { s.t. }\|\delta v\|=1 \\
\delta v \in V_{0}^{+}
\end{gathered}
$$

In case of adhesion, the second derivative of the potential energy is the sum of the elasticity stiffness operator $K$ and the second derivative of the adhesive potential $D$, which may be negative: $A=\frac{\partial^{2} E_{p}}{\partial u^{2}}=\frac{\partial^{2} E_{\text {elas }}}{\partial u^{2}}+\frac{\partial^{2} E_{a d h}}{\partial u^{2}}=K+D$. We point out that $K$ does not depend on the equilibrium solution whereas $D$ does. Expliciting the constraints of periodicity, zero average value and positivity over the contact region for the admissible perturbation direction $\delta v$, the previous problem becomes:

$$
\begin{aligned}
\lambda_{\min }=\min _{\delta v} & \frac{1}{2} \delta v \cdot A \cdot \delta v \\
\text { s.t. }\|\delta v\|=1 & \\
& \delta v \text { periodic } \\
& \int_{S} \delta v(\underline{x}) d S=0 \\
& \delta v(\underline{x}) \geqslant 0 \quad \forall \underline{x} \in I_{c}
\end{aligned}
$$

The previous problem is a non-standard eigenvalue problem since the last inequality constraints cannot be tackled by standard eigenvalue solvers. Although this is not the purpose of this work, we point out that this problem can be formulated as an eigenvalue analysis of a nonlinear operator and that many interesting properties of eigenvalues of linear operators are lost when dealing with non-linear operators. In particular, the set of eigenvalues is not necessarily discrete, or even countable, and it is not obvious how to orthogonalize eigenvectors.

Before discussing algorithmic issues for solving the previous problem, we first present the discrete version of the eigenvalue problem. The stability analysis in case of contact implies the computation of the minimal eigenvalue of the operator $\mathbf{A}=\mathbf{K}+\mathbf{D}$ under unilateral constraints $x_{i} \geqslant 0$ for $i \in I_{c}$ where $I_{c}$ is the contact zone defined from the equilibrium discrete solution $\mathbf{u}^{\mathbf{e q}}$ of problem (14) by:

$$
i \in I_{c} \Leftrightarrow u_{i}^{e q}-h_{i}=0
$$

and where $\mathbf{x}$ denotes a potential perturbation. We denote by $C=\left\{\mathbf{x}\right.$ such that $x_{i} \geqslant 0$ for $\left.i \in I_{c}\right\}$ the cone of unilateral constraints. We aim at solving the following eigenvalue problem:

$$
\begin{aligned}
\lambda_{\text {min }}=\min _{\mathbf{x}} & \frac{1}{2} \mathbf{x}^{\mathrm{T}} \mathbf{A} \mathbf{x} \\
\text { s.t. } & \|\mathbf{x}\|=1 \\
& \mathbf{e}^{\mathrm{T}} \mathbf{x}=0 \\
& \mathbf{x} \in C
\end{aligned}
$$

where the constraint $\mathbf{e}^{\mathrm{T}} \mathbf{x}=0$ imposes null mean value for the eigenvector (periodicity is imposed by construction of operator $\mathbf{A}$, see section 2.2).

3.2 Proximal algorithm for solving the constrained-eigenvalue problem

In the presence of contact, the associated eigenvalue problem (22) falls within the class of coneconstrained eigenvalue problems $[37,35,20,11]$. Determining all the possible solutions to such problems is challenging, although it is possible to enumerate all of them by solving $2^{n_{c}}$ standard eigenvalue problems followed by the verification of the corresponding constraints where $n_{c}$ 
is the number of contact constraints [11]. However, such a method cannot be used in practice because of the exponential growth of the number of systems to solve as a function of $n_{c}$. It has been shown that cone-constrained eigenvalue problems can be formulated as mixed non-linear complementarity problems $[9,8,20]$ for which various algorithms can be used such as non-smooth Newton or interior-point methods. However, these different approaches usually require to solve, at least, one linear system at each iteration which is too expensive in the present case because the linear system matrix $\mathbf{A}$ is dense. An alternative approach is to formulate the solution of the constrained eigenvalue problem as the minimization of the Rayleigh quotient over the constrained space $[10,11]$ as in $(26)$. Gradient descent-like algorithms can therefore be used to find a solution of the constrained eigenvalue problem. In particular, such methods do not necessarily need to solve linear systems.

Our proposed algorithm belongs to this last category and fits, more generally, in the framework of proximal algorithms [6,31], now widely used in the signal processing community. It bears similarities with the scaling and projection algorithm proposed in [11] and the projected steepest ascent of [35] since it also involves projection steps and scaling factors. Since the proposed approach relies on the use of proximal algorithms which have many variants, it is possible that the algorithms of $[35,11]$ could also be formulated in this framework. One main difference of the proposed approach is its ability to tackle additional constraint such as the linear constraint $\mathbf{e}^{\mathrm{T}} \mathbf{x}=0$ imposing a zero average. Finally, let us also mention that the use of proximal algorithms provides a natural extension to more complicated nonlinear constraints defining the cone $C$ if projection operations can be efficiently computed. For instance, a similar approach has been proposed in [14] for the computation of eigenvalues of singular operators that appear in plasticity. Contrary to our method, this approach imposes to solve a linear system at each iteration.

The approach we propose can be considered as an operator-splitting method which is usually used for optimizing the sum of convex functions. Problem (26) is then transformed into an unconstrained problem aiming at minimizing the sum of four functions $F+G_{1}+G_{2}+H$ where $F=\mathbf{1}_{\{\|\mathbf{x}\|=1\}}$ is the indicator of the unit sphere, $G_{1}=\mathbf{1}_{C}$ and $G_{2}=\mathbf{1}_{\left\{\mathbf{e}^{\mathrm{T}} \mathbf{x}=0\right\}}$ are the indicators of the cone $C$ and the hyperplane $\mathbf{e}^{\mathrm{T}} \mathbf{x}=0$ and finally $H(\mathbf{x})=\frac{1}{2} \mathbf{x}^{\mathrm{T}} \mathbf{A x} . G_{1}, G_{2}$ are convex and $H$ is convex and differentiable.

The primal-dual proximal algorithm, developed in [7], enables minimizing the sum of these four functions, in case $F$ is convex, by taking advantage of the regularity of $H$ :

$$
\begin{aligned}
& \mathbf{x}_{n+1}=\operatorname{prox}_{\tau F}\left(\mathbf{x}_{n}-\tau \nabla H\left(\mathbf{x}_{n}\right)-\tau \sigma\left(\mathbf{y}_{n}^{1}+\mathbf{y}_{n}^{2}\right)\right) \\
& \mathbf{y}_{n+1}^{i}=\mathbf{y}_{n}^{i}+2 \mathbf{x}_{n+1}-\mathbf{x}_{n}-\operatorname{prox}_{G_{i} / \sigma}\left(\mathbf{y}_{n}^{i}+2 \mathbf{x}_{n+1}-\mathbf{x}_{n}\right) \quad i=1,2
\end{aligned}
$$

where the proximal operator of a function $f$ is defined by

$$
\operatorname{prox}_{f}(x)=\underset{y}{\arg \min } f(y)+\frac{1}{2}\|x-y\|^{2}
$$

The previous algorithm converges to the global minimizer of $F+G_{1}+G_{2}+H$ if $\frac{1}{\tau}-\sigma \geqslant \beta / 2$ where $\beta$ is the Lipschitz's constant of $\nabla H$.

We propose to extend this algorithm to the case where $F=\mathbf{1}_{\{\|\mathbf{x}\|=1\}}$ is non convex. Here, since $F, G_{1}$ and $G_{2}$ are indicator functions, the proximal operators reduce to projection operators. The 
iterations then read:

$$
\begin{aligned}
& \tilde{\mathbf{x}}_{n+1}=\mathbf{x}_{n}-\tau \mathbf{A} \mathbf{x}_{n}-\tau \sigma\left(\mathbf{y}_{n}^{1}+\mathbf{y}_{n}^{2}\right) \\
& \mathbf{x}_{n+1}=\tilde{\mathbf{x}}_{n+1} /\left\|\tilde{\mathbf{x}}_{n+1}\right\| \\
& \mathbf{y}_{n+1}^{1}=\mathbf{y}_{n}^{1}+2 \mathbf{x}_{n+1}-\mathbf{x}_{n}-\operatorname{Proj}_{C}\left(\mathbf{y}_{n}^{1}+2 \mathbf{x}_{n+1}-\mathbf{x}_{n}\right) \\
& \mathbf{y}_{n+1}^{2}=\mathbf{y}_{n}^{2}+2 \mathbf{x}_{n+1}-\mathbf{x}_{n}-\operatorname{Proj}_{\left\{\mathbf{e}^{\mathrm{T}} \mathbf{x}=0\right\}}\left(\mathbf{y}_{n}^{2}+2 \mathbf{x}_{n+1}-\mathbf{x}_{n}\right)
\end{aligned}
$$

By writing $\mathbf{y}_{n}=\mathbf{y}_{n}^{1}$ and $z_{n}$ such that $\mathbf{y}_{n}^{2}=z_{n} \mathbf{e}$, we obtain:

$$
\begin{aligned}
\tilde{\mathbf{x}}_{n+1} & =\mathbf{x}_{n}-\tau \mathbf{A} \mathbf{x}_{n}-\tau \sigma\left(\mathbf{y}_{n}+z_{n} \mathbf{e}\right) \\
\mathbf{x}_{n+1} & =\tilde{\mathbf{x}}_{n+1} /\left\|\tilde{\mathbf{x}}_{n+1}\right\| \\
\mathbf{y}_{n+1} & =\left\langle\mathbf{y}_{n}+2 \mathbf{x}_{n+1}-\mathbf{x}_{n}\right\rangle_{-}^{I_{c}} \\
z_{n+1} & =z_{n}+\frac{1}{N} \mathbf{e}^{\mathrm{T}}\left(2 \mathbf{x}_{n+1}-\mathbf{x}_{n}\right)
\end{aligned}
$$

where we set $\langle\mathbf{x}\rangle_{-}^{I_{c}}=\left\{\begin{array}{ll}x_{i} & \text { for } x_{i} \leqslant 0 \text { and } i \in I_{c} \\ 0 & \text { otherwise }\end{array}\right.$.

Although there is no proof of convergence when $F$ is not convex, numerical tests show that for parameters $\tau, \sigma$ verifying $\frac{1}{\tau}-\sigma \geqslant \beta / 2$, the algorithm does converge to the smallest eigenvalue of $\mathbf{A}$ in case $I_{c}=\varnothing$ and to an admissible solution $\mathbf{x} \in C$ in case $I_{c} \neq \varnothing$. This will be illustrated in Subsection 4.1

It is worth noting that the proposed algorithm requires only matrix-vector product with operator A and local operations which can be easily parallelized.

We propose two complementary indicators for the convergence of the algorithm. $\epsilon$ defined by:

$$
\epsilon=\left|\mathbf{e}^{\mathrm{T}} \mathbf{x}\right|
$$

and $\eta$ defined as

$$
\eta=\frac{\left|z_{n}-z_{n-1}\right|}{\left|z_{n}\right|}
$$

The final proximal algorithm is presented in Algorithm 1. The algorithm will stop when the eigenvalue $z_{n}$ stagnates and when the condition $\mathbf{e}^{\mathrm{T}} \mathbf{x}_{n}=0$ is verified to a given tolerance.

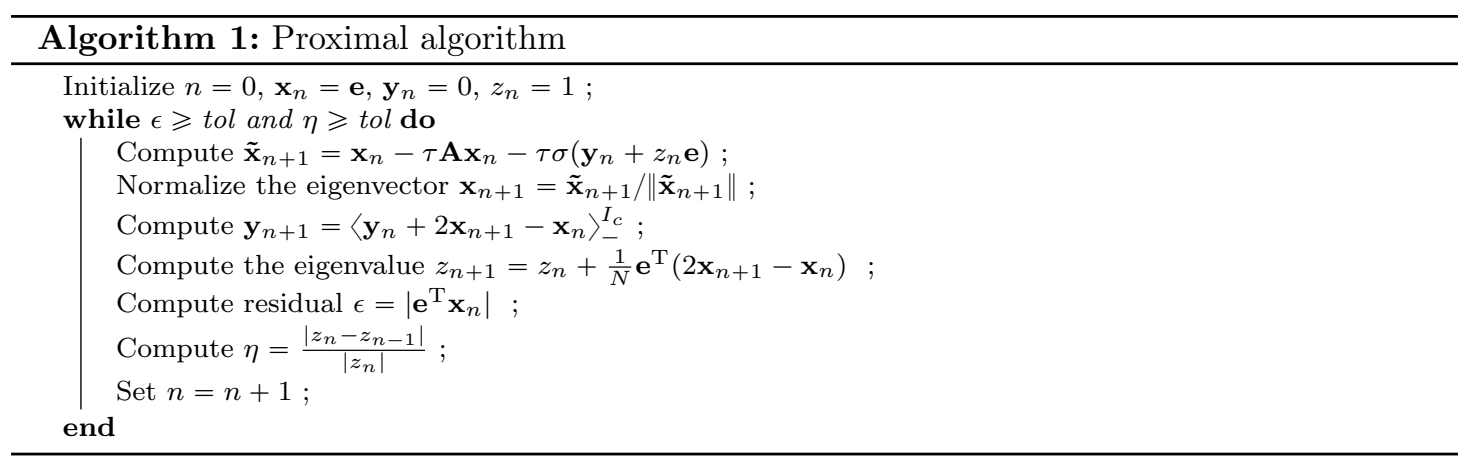

The choice of the scalar $\beta$ has a influence on the speed of convergence of the algorithm. 


\section{Numerical assessments}

First, we illustrate the proximal algorithm on the simple example of Hertzian contact. The computations are done on a coarse grid to ease the visualization of eigenvectors. We show that the instability modes depend on the geometry of the indenter. Then, we consider rough contact with three different potentials and observe different conclusions on stability depending on the potential.

\subsection{Adhesive Hertzian contact: influence of the curvature of the indenter}

The profile of the rigid rough surface is made of one asperity of parabolic shape:

$$
h(r)=-\frac{r^{2}}{2 R}
$$

with $R=2 L$ and $r=\sqrt{\left(x-\frac{L}{2}\right)^{2}+\left(x-\frac{L}{2}\right)^{2}}$. The origin of the system of axis is chosen such that it coincides with the top of the indenter. For this subsection, we consider the following adhesive potential

$$
E_{a d}=-\gamma \int_{S} \exp \left(\frac{-g}{\rho}\right) d S
$$

where $\gamma$ is the surface energy and $\rho$ the range of adhesion effects. This first potential models short-range adhesion, which is supposed to give results close to the solution of Maugis [24]. Note that the Maugis-Dugdale potential being not twice differentiable, the stability analysis cannot be performed on it. The force due to adhesion and the operator $D$ are:

$$
\frac{\partial E_{a d}}{\partial u}=\frac{\gamma}{\rho} \exp \left(\frac{-g(\underline{x})}{\rho}\right) \quad D=\frac{\partial^{2} E_{a d}}{\partial u^{2}}=-\frac{\gamma}{\rho^{2}} \exp \left(\frac{-g(\underline{x})}{\rho}\right)
$$

We choose $E=1, \gamma=10$ and $\rho=1 L$ and make simulations on a grid of size $32 \times 32$. Of course, this discretization is coarse and solving the problem with finer discretization is possible but we chose such a grid on this first example to ease the visualization of the instability mode.

First, we impose the mean value $u_{0}=\frac{1}{N} \sum_{i=0}^{N} u_{i}=+1.302 L$. The equilibrium solution is such that there is no contact, as illustrated on Figure 3a (the gap is everywhere strictly positive). We used the proximal algorithm to compute the smallest eigenvalue. We chose $\tau=1 / \widehat{\beta}, \sigma=\widehat{\beta} / 2$ and $\hat{\beta}=\sqrt{\|\mathbf{A}\|_{1}\|\mathbf{A}\|_{\infty}} \geqslant\|\mathbf{A}\|_{2}=\beta$. Let note that, in this case, since $I_{c}=\varnothing$, the problem is unconstrained so that traditional eigenvalue solver could be used. We obtain $\lambda_{\min }=-7.310^{-6}$ and the eigenvector is illustrated on Figure $3 \mathrm{~b}$. We verified that this solution is also the one found by a standard eigenvalue solver. We observe that adhesion tends to pull the elastic surface towards the indenter.

Now we consider the equilibrium solution obtained for the imposed mean displacement $u_{0}=$ 1.2L. The equilibrium solution, exhibiting a central contact zone, is illustrated on Figure 4a. We used the proximal algorithm with the same values for $\tau$ and $\sigma$ as for the previous case to compute the smallest eigenvalue under constraints. The algorithm converges to $\lambda_{\min }=-4.55$ that seems to be a multiple eigenvalue because of the problem symmetry. Since $\lambda_{\min }$ is negative, we can conclude that the equilibrium solution is not stable. The value of the eigenvector at $y=\frac{L}{2}$ is 


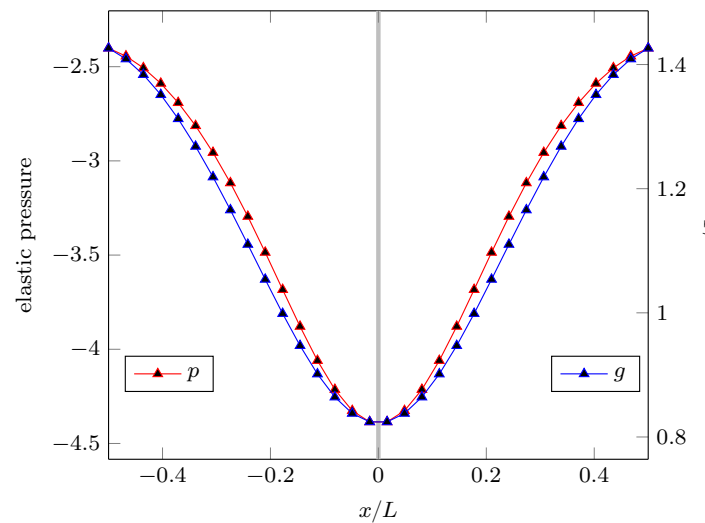

(a) equilibrium solution at $y=\frac{L}{2}$

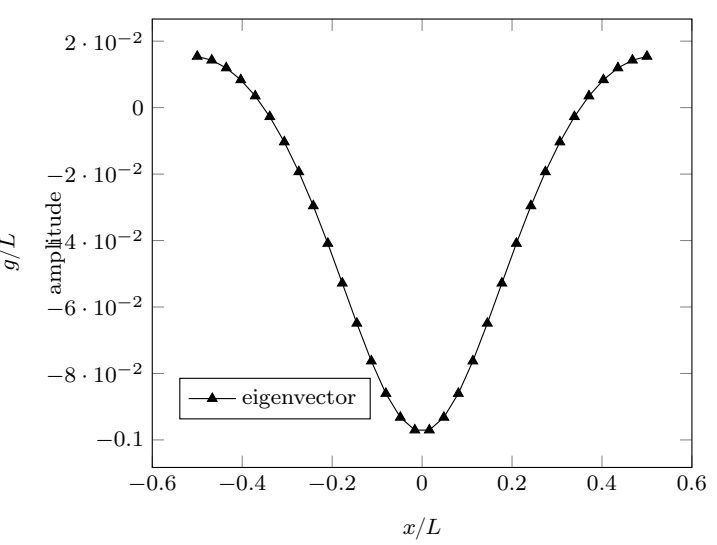

(b) eigenvector at $y=\frac{L}{2}$

Fig. 3: Hertz case: equilibrium solution for imposed displacement $u_{0}=+1.302 L$ (solution without contact) and instability mode associated to $\lambda_{\min }=-7.310^{-6}$

represented on Figure 4b. We observe that this vector is periodic, of zero average and positive in the contact zone, it is thus admissible with respect to $V_{0}^{+}$. We also note that this vector is associated to an instability of the points located at the contact zone boundary, where the gap between the two surfaces is the smallest (but still strictly positive). Therefore, slight perturbation of the applied load or imperfection would tend to make the two surfaces in contact at the contact zone boundary.

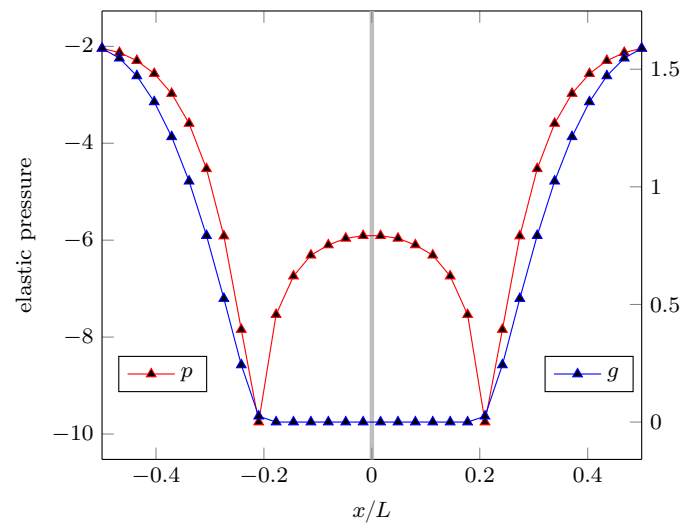

(a) equilibrium solution at $y=\frac{L}{2}$

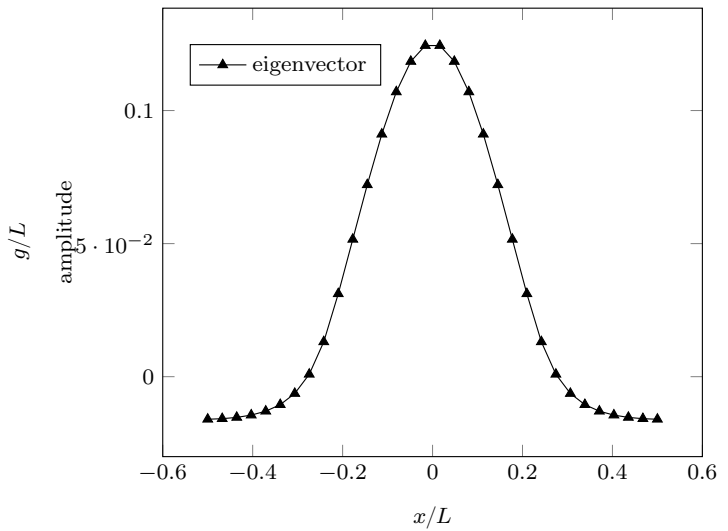

(b) eigenvector at $y=\frac{L}{2}$

Fig. 4: Hertz case: equilibrium solution for imposed displacement $u_{0}=1.2 L$ (solution with a contact zone) and instability mode associated to $\lambda_{\min }=-4.55$

In Figure 5, we give the evolution of the two quantities $\epsilon$ and $\eta$ during the iterations of the proximal algorithm for the two different imposed displacements: on Figure 5a for $u_{0}=1.302 \mathrm{~L}$ (where the equilibrium solution leads to no contact) and on Figure $5 \mathrm{~b}$ for $u_{0}=1.2 \mathrm{~L}$. 


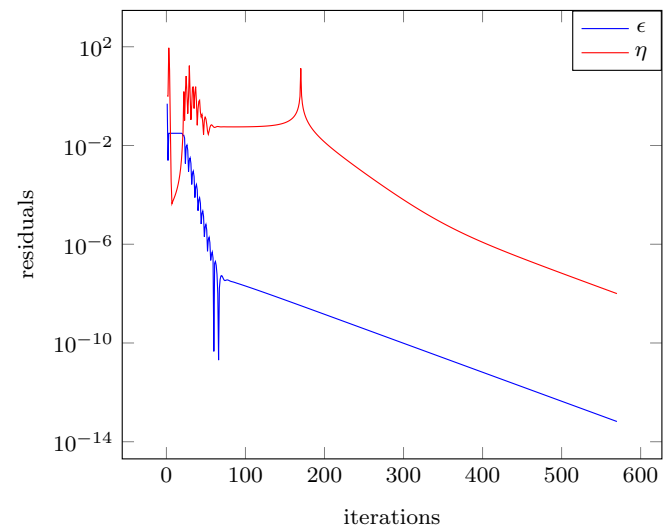

(a) No contact zone $\left(I_{c}=\varnothing\right)$

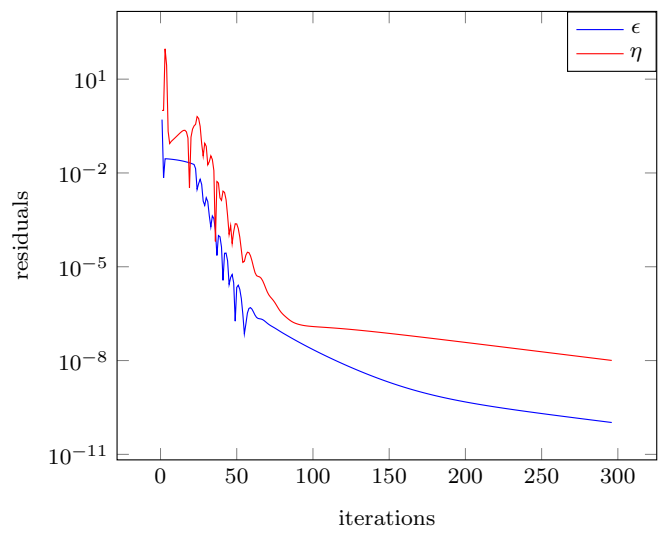

(b) Contact zone $\left(I_{c} \neq \varnothing\right)$

Fig. 5: Residuals $\eta$ and $\epsilon$ versus iterations of the proximal algorithm for the two imposed displacements $u_{0}=1.302 L$ and $u_{0}=1.2 L$

\subsection{Adhesive rough contact}

In this subsection, we consider the adhesive normal contact with rough surface. After describing the rough surface, we present results for three different potentials.

\subsubsection{Description of the rough surface}

As mentioned in the introduction, surface are rough and made of multiple asperities. Rough surface is usually modeled as an isotropic Gaussian random field with mean zero and with specified power spectral density (PSD), which is the Fourier transform of the auto-correlation function $[23]$.

In order to give the form of the PSD we will consider in this paper, we introduce the wavelengths: $\lambda_{s}$ (shortest wavelength), $\lambda_{r}$ (roll-off wavelength) and $\lambda_{l}$ (largest wavelength). As a consequence, we can defined the associated wave numbers: $k_{s}=\frac{L}{\lambda_{s}}, k_{r}=\frac{L}{\lambda_{r}}$ and $k_{l}=\frac{L}{\lambda_{l}}$.

The power spectral density of natural rough surfaces usually follows a self-affine behavior [23] so that the PSD reads:

$$
\Phi(|\mathbf{k}|)= \begin{cases}C & \text { if } k_{l} \leqslant|\mathbf{k}| \leqslant k_{r} \\ C\left(\frac{|\mathbf{k}|}{k_{r}}\right)^{-2-H} & \text { if } k_{r} \leqslant|\mathbf{k}| \leqslant k_{s} \\ 0 & \text { otherwise }\end{cases}
$$

where $H$ is the Hurst exponent and $C$ the roughness amplitude. $|\mathbf{k}|$ is the norm of the wave vector:

$$
|\mathbf{k}|=\sqrt{k_{x}^{2}+k_{y}^{2}}
$$

To construct periodic rough surfaces, we use a Fourier-based filtering algorithm [15]. We used this filtering process to generate one $256 \times 256$ rough surface with the following properties $L=1, k_{l}=2, k_{r}=2, k_{s}=32, H=0.8$. The width of the spectrum is quite small and may not represent the self-affinity of the surface. Indeed, we chose to reduce the spectrum so that obtaining a sufficient discretization for adhesive contact was possible with a grid $256 \times 256$.

In figure 6 , we give a representation of the rough surface. Since this surface corresponds to one realization of the random Gaussian surface with defined cutoff wave numbers and Hurst 
exponent, one cannot conclude anything on the influence of the wavelengths on the mechanical response. Indeed, such observations would require statistically converged results, that is to say many surface realizations. As it is not the objective of this paper, we will only perform stability analysis for this specific surface.

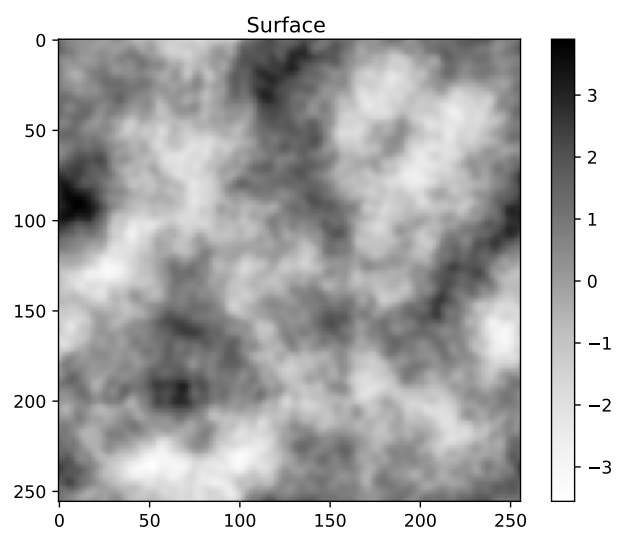

Fig. 6: Heights of the rough surface

\subsubsection{Stability analysis for three different potentials}

Here we will consider three adhesive potentials that were studied in [28,27] and defined as follows:

$$
\begin{gathered}
E_{a d h 1}=-\gamma \int_{S} \exp \left(\frac{-g}{\rho}\right) d S \\
E_{a d h 2}=-\gamma \int_{S} \exp \left(\frac{-g^{2}}{2 \rho^{2}}\right) d S \\
E_{a d h 3}=-\gamma \int_{S} \frac{1}{1+\frac{g^{2}}{\rho^{2}}} d S
\end{gathered}
$$

In Figure 7a, we give the graphical representation of those three potentials and in Figure 7b, we plot the respective associated force, which is the gradient of the potential. The plots presented on Figure 7 are presented for $\rho=0.5 L$ and $\gamma=0.01$. With the first potential $E_{a d h 1}$ the adhesion forces are not zero when the distance between the two surfaces is zero. This is not the case for the second and the third adhesive potentials $E_{a d h 2}$ and $E_{a d h 3}$. The third potential may represent the long-range Van der Waals interactions.

For this computation, we choose $E=1, \gamma=1$ and $\rho=0.1 L$ and compute the equilibrium solution with imposed mean value of displacement $u_{0}=0.8 \mathrm{~L}$ for the first potential, $u_{0}=0.105 \mathrm{~L}$ for the second potential and $u_{0}=0.65 \mathrm{~L}$ for the third potential. The three adhesive potentials lead to same relative contact area $a_{c}=0.23$ but to different contact pattern, as evidenced by the map of the binary gap between the two surfaces at the equilibrium configuration represented on Figure 8 . 


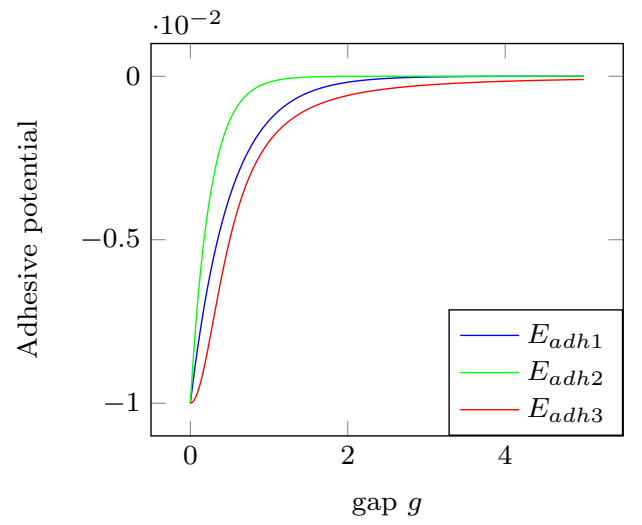

(a) Adhesive potentials

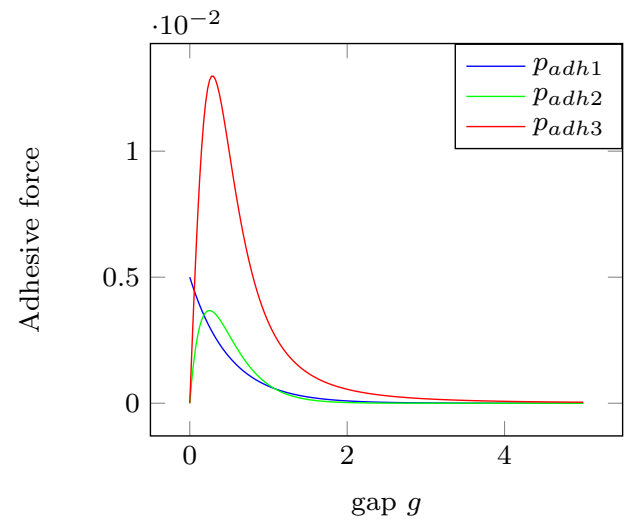

(b) Adhesive forces

Fig. 7: Graphical representation of the three adhesive potentials and associated forces

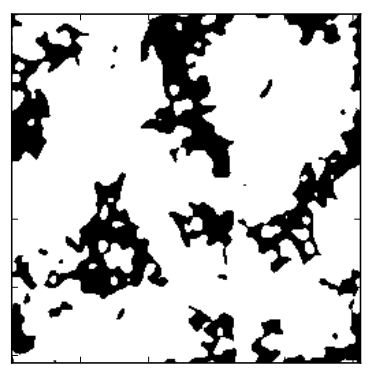

$E_{\text {adh } 1}$

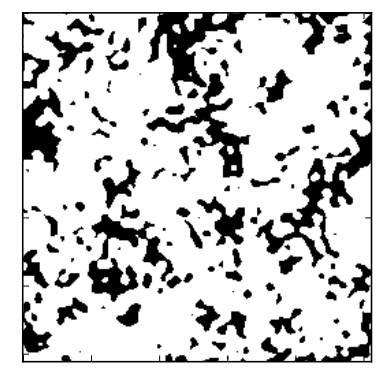

$E_{a d h 2}$

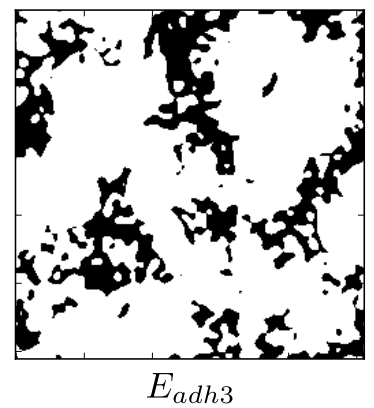

Fig. 8: Equilibrium solution : gap obtained for the three adhesive potentials: black means contact, white means no contact.

The stability analysis is then performed and the three eigenvectors associated to the three eigenvalues are represented on Figure 9. We verify that those eigenvectors are such that the non interpenetration constraint (last inequality of (24)) is verified.

With the stability analysis, we can conclude that the equilibrium solutions obtained with adhesive potential 1 and 2 are unstable (the eigenvalues are negative). The associated eigenvector are different. The equilibrium solution computed in the case of the Van de Waals potential is stable since the eigenvalue is positive.

For the second computation, we choose $E=1, \gamma=1$ and $\rho=0.1 L$ and compute the equilibrium solution with imposed mean value of displacement $u_{0}=0.105 L$ for all the potentials. The three adhesive potentials lead different contact area, as evidenced by the map of the binary gap between the two surfaces at the equilibrium configuration represented on Figure 10.

The stability analysis is then performed and the three eigenvectors associated to the three eigenvalues are represented on Figure 11. We verify that those eigenvectors are such that the non interpenetration constraint (last inequality of (24)) is verified.

As for the previous case, only the third equilibrium solution (associated to the adhesive potential $E_{a d h, 3}$ is stable and the instability modes are not similar for the two other potentials. 

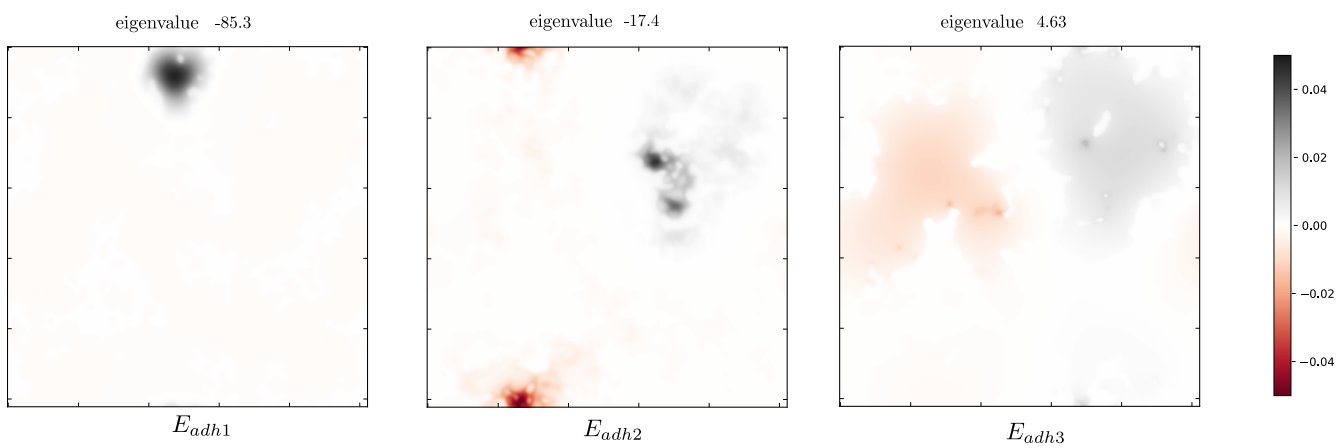

Fig. 9: Eigenvectors corresponding to the smallest eigenvalue of the constrained eigenvalue problem for the three adhesive potentials.
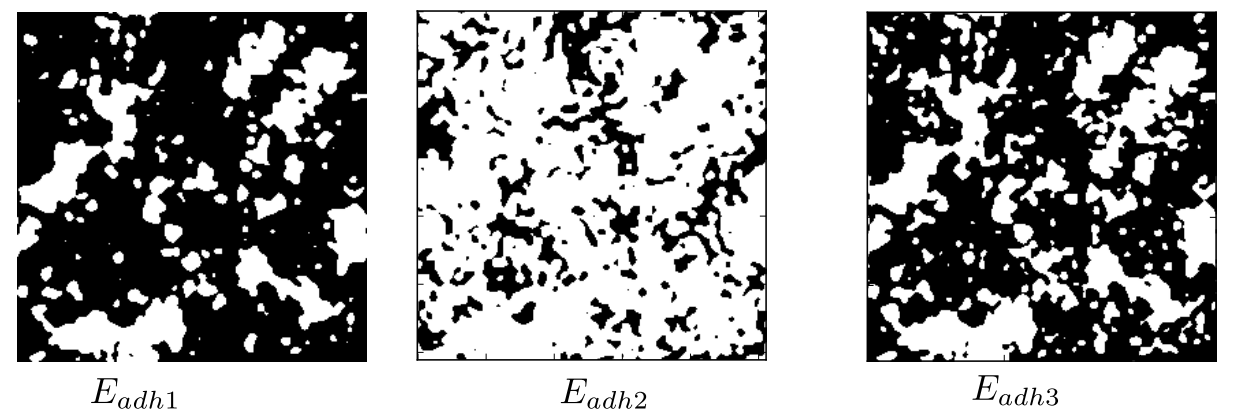

Fig. 10: Equilibrium solution : gap obtained for the three adhesive potentials: black means contact, white means no contact.
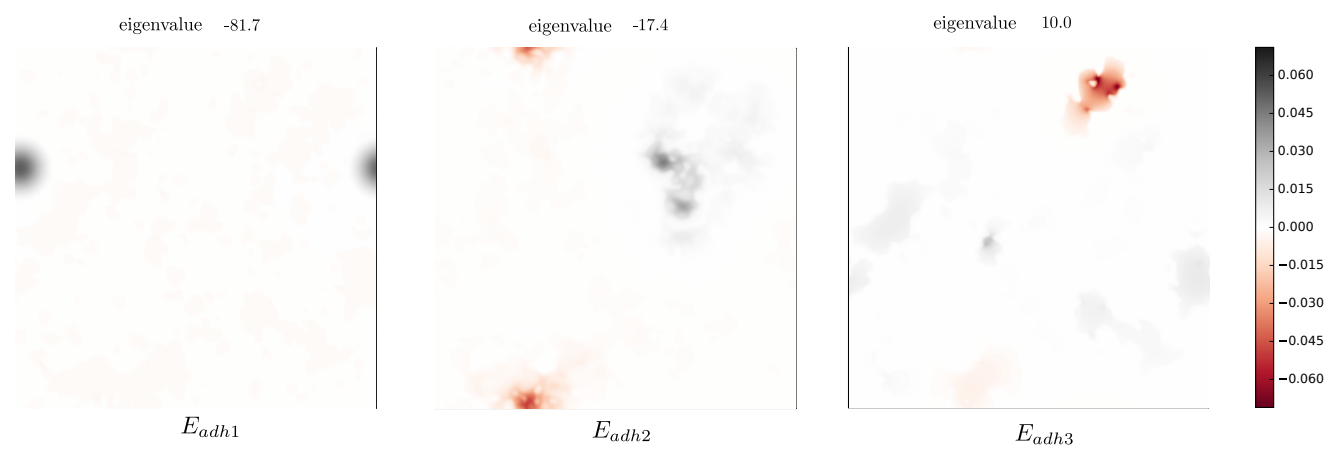

Fig. 11: Eigenvectors corresponding to the smallest eigenvalue of the constrained eigenvalue problem for the three adhesive potentials.

\subsubsection{Stability analysis during detachment}

As a final numerical example, we present the evolution of the equilibrium solution and its stability from almost full contact to full detachment. We use the same rough surface, the adhesive potential $E_{a d h 1}$ with parameters $E=10^{-3}, \gamma=10^{-2}$ and $\rho=1.0 \mathrm{~L}$ and impose mean values of the displacement $u_{0}$ from $u_{0}=10^{-5} L$ (which corresponds to almost full contact) to $u_{0}=(2+$ 
$\left.10^{-5}\right) L$. To compute the equilibrium solution, we use the approach presented in [36] and choose as initialization of the iterative solver the converged solution of the previous load. For each equilibrium solution, we compute the percentage of area that is is contact, $a_{c}=\frac{1}{N} \sum_{i \in I_{c}} i$ and use the proximal algorithm to compute the eigenvalue solution of problem (26).

On Figure 12a, we give the evolution of the mean value of the elastic pressure $p_{0}=\frac{1}{N} \sum_{i=1}^{N} p_{i}$ versus the mean value of the applied displacement $u_{0}$ for all the computed equilibrium solutions. For each equilibrium solution, the stability analysis has been performed and the mark is full for stable positions and empty for unstable solutions. Figure $12 \mathrm{~b}$ gives the evolution of the contact area $a_{c}=\frac{1}{N} \sum_{i \in I_{c}} i$ versus $u_{0}$.

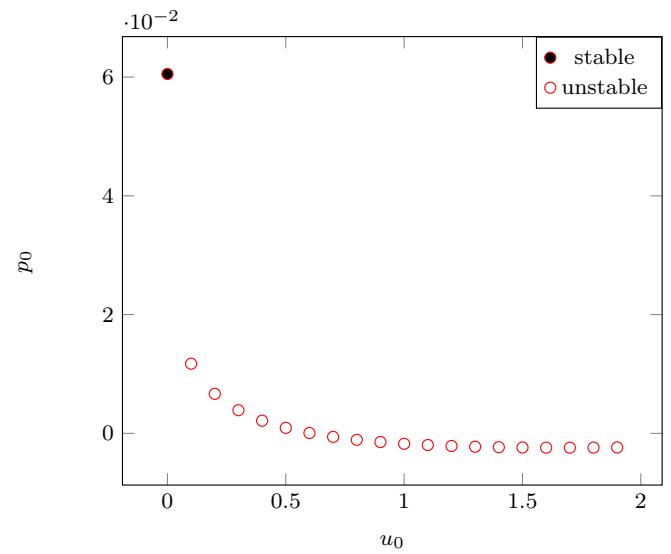

(a) Mean elastic pressure $p_{0}$ versus imposed displacement $u_{0}$ for $\rho=1 L$

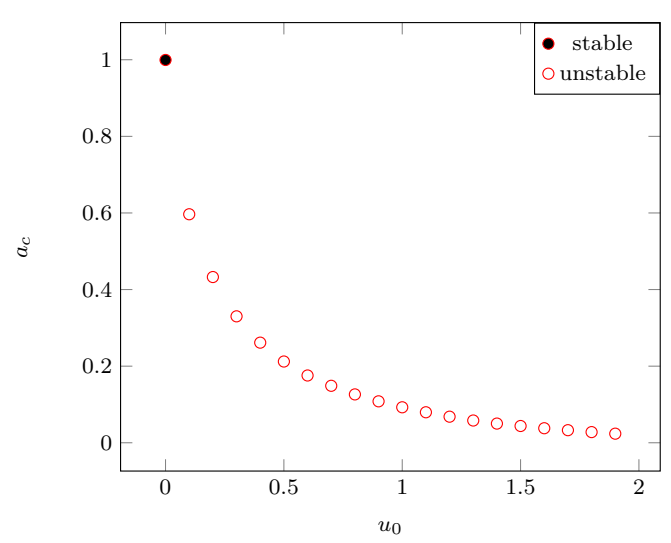

(b) Contact zone $a_{c}$ versus imposed displacement $u_{0}$ for $\rho=1 L$

Fig. 12: Mean elastic pressure $p_{0}$ and contact zone $a_{c}$ versus imposed mean displacement $u_{0}$ for $\rho=1 L:$ stable positions are with full circle

Finally, we set $\rho=0.1 L$ and do the same unloading and stability study. On Figure 13a, we give the evolution of the mean value of the elastic pressure $p_{0}$ versus the applied displacement $u_{0}$ for all the computed equilibrium solutions and specify the stability with a full mark.

We observe that contrary to the previous case, only one equilibrium is unstable. This is due to the ambivalent role of $\rho$ on adhesion: on the one hand the range of adhesive interactions is smaller, on the other hand, adhesive forces are larger at small distances. This explains why the number of unstable configurations is smaller than for the previous case and also why the first unstable configuration occurs at smaller $u_{0}$ than for the previous case.

\section{Conclusion}

In this article, we propose a proximal algorithm that enables the systematic study of stability of surfaces in presence of adhesion. This algorithm can be used for any type of surface (including rough surfaces) and any twice differentiable adhesive potential. It computes the smallest eigenvalue and associated eigenvectors so that the equilibrium solution can be discriminated as stable or unstable and in case of instability, the eigenvector show the associated mode. Future work will 


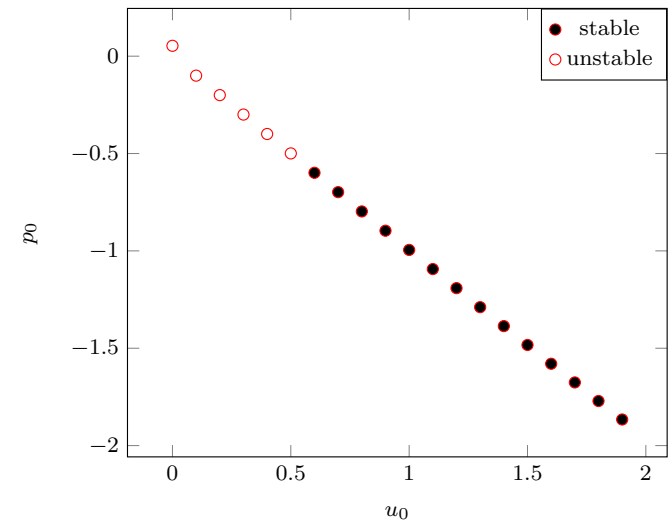

(a) Mean elastic pressure $p_{0}$ versus imposed displacement $u_{0}$ for $\rho=0.1 L$

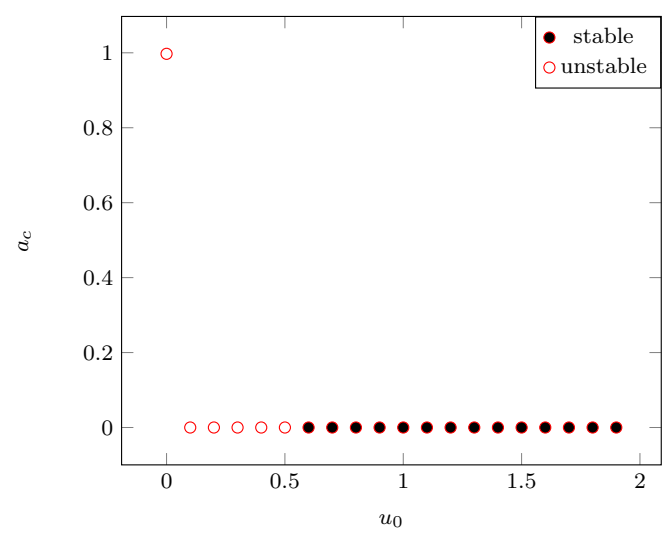

(b) Contact zone $a_{c}$ versus imposed displacement $u_{0}$ for $\rho=0.1 L$

Fig. 13: Mean elastic pressure $p_{0}$ and contact zone $a_{c}$ versus imposed mean displacement $u_{0}$ for $\rho=0.1 L$ : stable positions are with full circle

consist in studying loading/unloading curve by following a path of stable equilibrium solutions and expose the hysteresis effect induced by adhesion. It is important to point out that the present work does not resolve the question of following a sequence of stable solutions along a loading path. Continuation methods or stochastic gradient descent may be appropriate candidates for achieving this objective.

\section{Acknowledgments}

Support for V.R. from the EPFL Fellows fellowship program co-funded by Marie SkłodowskaCurie, Horizon 2020 Grant agreement no. 665667 is gratefully acknowledged. The authors would like to thank Professor Jean-François Molinari and Guillaume Anciaux for helpful discussions.

\section{References}

1. Carbone, G., Mangialardi, L.: Adhesion and friction of an elastic half-space in contact with a slightly wavy rigid surface. Journal of the Mechanics and Physics of Solids 52(6), 1267 - 1287 (2004)

2. Carbone, G., Mangialardi, L.: Analysis of the adhesive contact of confined layers by using a Green's function approach. Journal of the Mechanics and Physics of Solids 56(2), $684-706$ (2008)

3. Carbone, G., Scaraggi, M., Tartaglino, U.: Adhesive contact of rough surfaces: Comparison between numerical calculations and analytical theories. European Physical Journal E 30, 65-74 (2009)

4. Carpick, R.W., Ogletree, D.F., Salmeron, M.: A general equation for fitting contact area and friction versus load measurements. Journal of Colloid and Interface Science 211, 395-400 (1978)

5. Chateau, X., Nguyen, Q.: Buckling of elastic structures in unilateral contact. European Journal of Mechanics - A/Solids 10(1), 71-89 (1991)

6. Combettes, P.L., Pesquet, J.C.: Proximal splitting methods in signal processing. In: Fixed-point algorithms for inverse problems in science and engineering, pp. 185-212. Springer (2011)

7. Condat, L.: A primal-dual splitting method for convex optimization involving Lipschitzian, proximable and linear composite terms. Journal of Optimization Theory and Applications 158(2), 460-479 (2013)

8. da Costa, A.P., Martins, J., Figueiredo, I., Júdice, J.: The directional instability problem in systems with frictional contacts. Computer Methods in Applied Mechanics and Engineering 193(3), 357-384 (2004)

9. Da Costa, A.P., Figueiredo, I., Júdice, J., Martins, J.: A complementarity eigenproblem in the stability analysis of finite dimensional elastic systems with frictional contact. In: Complementarity: applications, algorithms and extensions, pp. 67-83. Springer (2001) 
10. Da Costa, A.P., Seeger, A.: Numerical resolution of cone-constrained eigenvalue problems. Computational \& Applied Mathematics 28(1), 37-61 (2009)

11. Da Costa, A.P., Seeger, A.: Cone-constrained eigenvalue problems: theory and algorithms. Computational Optimization and Applications 45(1), 25-57 (2010)

12. Derjaguin, B., Muller, V., Toporov, Y.: Effect of contact deformations on the adhesion of particles. Journal of Colloid and Interface Science 53(2), $314-326$ (1975)

13. Dong, C., Bonnet, M.: An integral formulation for steady-state elastoplastic contact over a coated half-plane. Computational mechanics 28(2), 105-121 (2002)

14. Glowinski, R.: Variational Methods for the Numerical Solution of Nonlinear Elliptic Problems. SIAM (2015)

15. Hu, Y.Z., Tonder, K.: Simulation of 3-D random rough surface by 2-D digital filter and Fourier analysis. International Journal of Machine Tools and Manufacture 32, 83-90 (1992)

16. Jacq, C., Nelias, D., Lormand, G., Girodin, D.: Development of a three-dimensional semi-analytical elasticplastic contact code. Journal of Tribology 124(4), 653-667 (2002)

17. Jin, F., Guo, X.: Mechanics of axisymmetric adhesive contact of rough surfaces involving power-law graded materials. International Journal of Solids and Structures 50(20), 3375-3386 (2013)

18. Johnson, K.: The adhesion of two elastic bodies with slightly wavy surfaces. International Journal of Solids and Structures 32(3-4), 423-430 (1995)

19. Johnson, K.L.: Contact mechanics. Cambridge University press (1994)

20. Júdice, J.J., Sherali, H.D., Ribeiro, I.M.: The eigenvalue complementarity problem. Computational Optimization and Applications 37(2), 139-156 (2007)

21. K. L. Johnson, K.K., Roberts, A.D.: Surface energy and the contact of elastic solids. Proceedings of the Royal Society of London A 324(1558), 301-313 (1971)

22. Kesari, H., Lew, A.J.: Effective macroscopic adhesive contact behavior induced by small surface roughness. Journal of the Mechanics and Physics of Solids 59(12), 2488-2510 (2011)

23. Longuet-Higgins, M.S.: Statistical properties of an isotropic random surface. Philosophical Transactions of the Royal Society A 250(975), 157-174 (1957)

24. Maugis, D.: Adhesion of spheres: The JKR-DMT transition using a Dugdale model. Journal of Colloid and Interface Science 150(1), 243-269 (1992)

25. Maugis, D., Barquins, M.: Adhesive contact of sectionally smooth-ended punches on elastic half-spaces: theory and experiment. Journal of Physics D: Applied Physics 16(10), 1843 (1983)

26. Meakin, P.: Fractals, Scaling and Growth Far from Equilibrium. Cambridge University Press (1998)

27. Müser, M.H.: Single-asperity contact mechanics with positive and negative work of adhesion: Influence of finite-range interactions and a continuum description for the squeeze-out of wetting fluids. Beilstein Journal of Nanotechnology 5, 419-437 (2014)

28. Müser, M.H., Dapp, W.B.: The contact mechanics challenge: Problem definition. arXiv preprint arXiv:1512.02403 (2015)

29. Nguyen, Q.S.: Stability and nonlinear solid mechanics. Wiley (2000)

30. Papangelo, A., Ciavarella, M.: A Maugis Dugdale cohesive solution for adhesion of a surface with a dimple. Journal of The Royal Society Interface 14(127), 2016.0996 (2017)

31. Parikh, N., Boyd, S., et al.: Proximal algorithms. Foundations and Trends $\mathbb{R}$ in Optimization 1(3), 127-239 (2014)

32. Pastewka, L., Robbins, M.O.: Contact between rough surfaces and a criterion for macroscopic adhesion. Proceedings of the National Academy of Sciences 111(9), 3298-3303 (2014)

33. Prokopovich, P., Perni, S.: Multiasperity contact adhesion model for universal asperity height and radius of curvature distributions. Langmuir 26(22), 17,028-17,036 (2010)

34. Putignano, C., Afferrante, L., Carbone, G., Demelio, G.: A new efficient numerical method for contact mechanics of rough surfaces. International Journal of Solids and Structures 42, 338-343 (2012)

35. Queiroz, M., Judice, J., Humes Jr, C.: The symmetric eigenvalue complementarity problem. Mathematics of Computation 73(248), 1849-1863 (2004)

36. Rey, V., Anciaux, G., Molinari, J.F.: Normal adhesive contact on rough surfaces : efficient algorithm for FFT-based BEM resolution. Computational Mechanics pp. n/a-n/a (accepted manuscript, 2017)

37. Seeger, A.: Eigenvalue analysis of equilibrium processes defined by linear complementarity conditions. Linear Algebra and its Applications 292(1-3), 1-14 (1999)

38. Stanley, H.M., Kato, T.: An FFT-based method for rough surface contact. Journal of Tribology 119, 481-485 (1997)

39. Westergaard, H.: Bearing pressures and cracks. Journal of Applied Mechanics 6, 49-53 (1937)

40. Zhang, W., Jin, F., Zhang, S., Guo, X.: Adhesive contact on randomly rough surfaces based on the doublehertz model. Journal of Applied Mechanics 81(5), 051,008 (2014) 